\title{
Influence of elements thickness of separation devices on the finely dispersed particles collection efficiency
}

\author{
Andrey V. Dmitriev ${ }^{1, *}$, Vadim E. Zinurov ${ }^{1}$, and Oksana S. Dmitrieva ${ }^{2}$ \\ ${ }^{1}$ Kazan State Power Engineering University, 420066, Kazan, Krasnoselskaya str., 51, Russia \\ ${ }^{2}$ Kazan National Research Technological University, 420015, Kazan, Karl Marx str., 68, Russia
}

\begin{abstract}
This article presents a rectangular separator, developed by the authors, for purification of a multiphase gas flow from the finely dispersed particles with a diameter of up to $10 \mu \mathrm{m}$. In order to increase the separation process efficiency, a research was conducted to determine the optimum thickness of I-beam elements within the separator at different values of the flow inlet rate and length of I-beam elements. It was found that change in the wall thickness of elements leads to a change in the flow structure, which causes a decrease in the dust particles collection efficiency.
\end{abstract}

\section{Introduction}

Separation of a multiphase gas flow from the finely dispersed solid particles with a diameter of up to $10 \mu \mathrm{m}$ is a relevant objective at the present day. Devices, collecting the dust particles from the gas flows, cope with this task extremely inefficiently. Such devices include various modifications of cyclonic separators, wet dust collectors, filters, electric filters, etc. It should be noted that these devices are quite common for all industry fields due to the highly efficient separation of gas flows from particles larger than $30 \mu \mathrm{m}$. However, when collection of the dust particles smaller than $10 \mu \mathrm{m}$ is required, the efficiency of cyclonic separators decreases significantly [1-6]. The use of wet scrubbers involves the wastewater pollution, which leads to the need to purify the liquid and, as a consequence, to a significant increase in the cost of gas separation process. The use of filters for this purpose is also economically inappropriate due to the need for frequent replacement of expensive filter elements, caused by abrasiveness of solid particles [7, 8]. The use of electric filters is not always possible, since the collected particles can be explosive. Also, serious limitations, narrowing the field of use of dry electric filters, are the inability to achieve stable residual dust content below $50 \mathrm{mg} / \mathrm{m}^{3}$ inside of them without a significant increase in costs for cleaning and insufficient collection efficiency at high electrical resistivity of dust [9-12].

In previous works [13] the authors of this article presented the developed rectangular separator for collecting the finely dispersed solid particles of less than $10 \mu \mathrm{m}$. This device is a rectangular case with dimensions $a \times c \times h$. There are several rows of elements inside of

\footnotetext{
*Corresponding author: ieremiada@gmail.com
} 
the device. When a gas flow passes through the separator, a centrifugal force arises between the device elements, discarding the particles to the walls of elements and ejecting them from the structural flow. The previously conducted research showed that the gas flow separation efficiency from dust particles of up to $10 \mu \mathrm{m}$ by means of the rectangular separator was on average by $50 \%$ higher than by means of the cyclonic separator TSN-11400 . When collecting the particles of 10-100 $\mu \mathrm{m}$, efficiency of both devices was on average $99.7 \%$. The pressure losses within the rectangular separator were $360 \mathrm{~Pa}$ against $540 \mathrm{~Pa}$ within the cyclonic separator at a volumetric air flow inlet rate of $0.05 \mathrm{~m}^{3} / \mathrm{s}$. Various design elements of a rectangular separator were also considered. The greatest efficiency and the lowest hydraulic resistance within the device are achieved by using the I-beam elements.

It is most advisable to use a rectangular separator as the second stage of cleaning system at the enterprise after a cyclonic separator or other dust collector with an efficiency of $99.9 \%$ from solid particles larger than $30 \mu \mathrm{m}$.

\section{Purpose and methods of research}

In order to increase the efficiency of collecting the finely dispersed particles and reduce the hydraulic resistance of the separator, it is necessary to determine the optimum structural dimensions of the device elements. The purpose of this work is to determine the optimum thickness of I-beam elements within a rectangular separator.

The research was conducted for a simplified model of a rectangular separator. Fig. 1 shows the first stage of the rectangular separator.

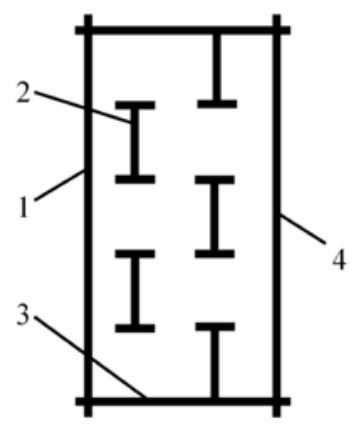

Fig. 1. The simplified two-dimensional model of a rectangular separator (top view): 1 - inlet nozzle, connecting the gas pipeline with the rectangular separator; 2 - I-beam element within the separator; 3 - device body; 4 - outlet nozzle.

The previously conducted research shows that the I-beam elements within the separator shall be arranged to meet the following condition:

$$
h_{1}=0.25 b,
$$

where $h_{1}$ - length of the I-beam element's projection, $\mathrm{m} ; b$ - length of the I-beam element, m.

A dependency was also obtained in order to determine the distance between the extreme starting points of the projections of I-beam elements between adjacent rows:

$$
l=0.625 b
$$

It should be noted that the arrangement of I-beam elements within the separator as per the formula (2) allows achieving the maximum value of the centrifugal force, affecting the separated flow, which significantly increases the efficiency of device. This dependency was 
obtained as a result of the following judgment - the circle, drawn from the center of I-beam element shall pass through the extreme points of the projections of I-beam elements of neighbor rows.

The finite element method in ANSYS Fluent software was used in order to determine the optimum thickness of I-beam elements within the separator. In the course of research, the turbulence model - SST was used. In the course of this research, the following invariant parameters were set: atmospheric outlet pressure was $10^{5} \mathrm{~Pa}$; initial rate of particles in the gas flow was $0 \mathrm{~m} / \mathrm{s}$; number of particles in the gas was $n=1000$; mass flow rate of combustion products was $G=10 \mathrm{~g} / \mathrm{s}(0.01 \mathrm{~kg} / \mathrm{s})$.

In order to obtain the results, the following parameters were changed: gas inlet rate $W$ was in the range of $3-7 \mathrm{~m} / \mathrm{s}$; diameter of the particles in the gas was in the range of $a=1-12$ $\mu \mathrm{m}$; length of I-beam elements $b$ was taken equal to 10,14 and $20 \mathrm{~mm}$; wall thickness $\delta$ was varied in the range of $0.5-1.5 \mathrm{~mm}$. In order to obtain the dependencies between them, one of the parameters was varied in the specified range of values, but other parameters were set to the basic values. Basic values: gas inlet rate was $W=5 \mathrm{~m} / \mathrm{s}$, diameter of particles was $a=5 \mu \mathrm{m}$, length of I-beam elements was $b=14 \mathrm{~mm}$, thickness of walls was $\delta=1 \mathrm{~mm}$.

Numerical calculation was carried out under the following assumptions: gas flow process was steady-state, dust concentration excluded the interaction between particles, influence of particles on the carrying medium movement was not considered.

In the course of this research the efficiency of the first stage of a rectangular separator was determined as follows:

$$
E=1-\frac{n_{k}}{n}
$$

where $n_{k}$ - number of particles, remained in the gas flow after the separation process within device,

and the hydraulic resistance coefficient of the rectangular separator was determined as follows:

$$
\xi=\Delta p \frac{l}{h} \frac{2}{\rho w_{h}^{2}},
$$

where $\Delta p$ - pressure losses within the separator, $\mathrm{Pa} ; h$ - distance between the first and the last rows of I-beam elements, $\mathrm{m} ; \rho-$ gas density, $\mathrm{kg} / \mathrm{m}^{3} ; w_{h}-$ gas rate within the constrictions of a rectangular separator, $\mathrm{m} / \mathrm{s}$. In this study values $l$ and $h$ are equal.

Rate within the constrictions of a rectangular separator can be determined by the formula:

$$
w_{h}=\frac{16}{3} W
$$

\section{Research results and discussion of them}

Research results are presented graphically in Fig. 2-6. Numerical studies show that the thickness of I-beam walls significantly affects the gas flow separation efficiency. Due to the changes in the thickness of walls, the curvature of the narrowing space between I-beam elements also changes, this entails a change in the flow structure. It was found that the maximum gas flow separation efficiency is achieved at the wall thickness $\delta=1 \mathrm{~mm}$ (Fig. 2). The average flow separation efficiency by the first stage of the separator at a wall thickness of $1 \mathrm{~mm}$, is $43 \%$, which is by $15 \%$ and $22 \%$ higher than at a wall thicknesses of 
0.5 and $1.5 \mathrm{~mm}$, respectively. It should be noted that blowing the high rates of the gas flow by fan leads to a decrease in separation efficiency, due to rebound of dust particles back into the structural gas flow. However, on the other hand, when the gas flow rate increases, the centrifugal force also increases, which positively affects the separation. Thus, at the gas rate $W=5 \mathrm{~m} / \mathrm{s}$, the average separation efficiency is $40.5 \%$, which is higher by $23.5 \%$ and $5.1 \%$ than at the gas rates of 3 and $7 \mathrm{~m} / \mathrm{s}$, respectively. Therefore, control and analysis of the blowing gas flow rate is an important component to improve the separation efficiency.



Fig. 2. Change in the gas flow separation efficiency by the first stage of a rectangular separator from the thickness of I-beam walls at the following inlet gas flow rate $W, \mathrm{~m} / \mathrm{s}: 1-3 ; 2-5 ; 3-7$.

Due to the fact that at the gas flow rate $W=5 \mathrm{~m} / \mathrm{s}$ and the wall thickness $\delta=1 \mathrm{~mm}$, the maximum gas separation efficiency is achieved, and these values were taken as basic in the subsequent research. When the length of I-beam elements is $b=14 \mathrm{~mm}$ and thickness of walls is $1 \mathrm{~mm}$, efficiency of collecting the finely dispersed particles is $54.1 \%$, which is the highest value in the course of studies of changes in the length of I-beam elements (Fig. 3).



Fig. 3. Change in the gas flow separation efficiency by the first stage of a rectangular separator from the thickness of I-beam walls at the following thickness of I-beam elements $b, \mathrm{~mm}: 1-10 ; 2-14 ; 3-$ 20 .

When the length of I-beam elements is $14 \mathrm{~mm}$, the average gas separation efficiency is $40.5 \%$, which is by $13.7 \%$ and $15.6 \%$ higher than when the lengths of I-beam elements is 10 and $20 \mathrm{~mm}$, respectively. It should be noted that when the length of I-beam elements is more than $15 \mathrm{~mm}$, thickness of the walls shall be increased in order to improve the efficiency of gas separation process (Fig. 3). An increase in the diameter of particles in the separated gas leads to an increase in the gas flow separation efficiency (Fig. 4). Average efficiency of the dust particles collection is $16.6 \%, 40.5 \%$ and $71.6 \%$ with a diameter of 1 , 5 and $9 \mu \mathrm{m}$, respectively. 


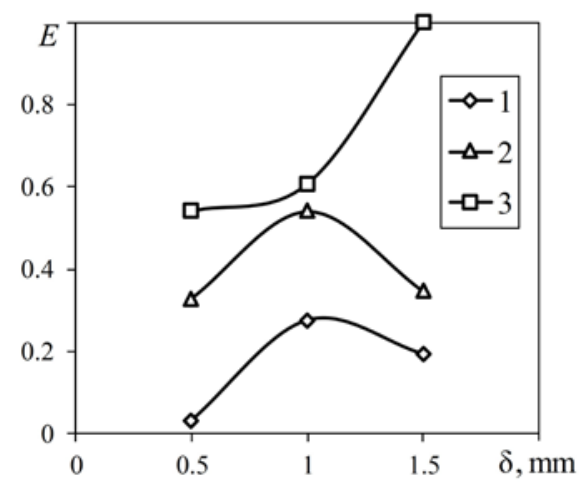

Fig. 4. Change in the gas flow separation efficiency by the first stage of a rectangular separator from the thickness of I-beam walls at the following diameter of particles $a, \mu \mathrm{m}: 1-1 ; 2-5 ; 3-9$.

It should be noted that when collecting the particles of up to $8 \mu \mathrm{m}$, the maximum separation efficiency is observed at a thickness of I-beam walls $\delta=1 \mathrm{~mm}$, however, when separating the gas from particles with a diameter of more than $8 \mu \mathrm{m}$, the greatest efficiency is observed at the walls with thickness of $1.5 \mathrm{~mm}$ (Fig. 5).

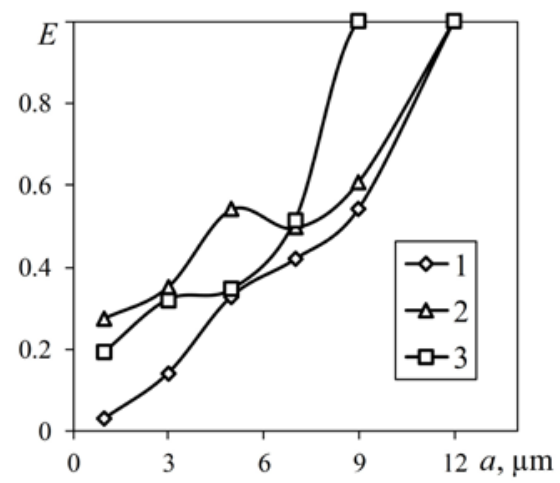

Fig. 5. Change in the gas flow separation efficiency by the first stage of a rectangular separator from the diameter of particles at the following thickness of I-beam elements $\delta$, mm: $1-0.5 ; 2-1 ; 3-1.5$.

Moreover, when the diameter of dust particles is more than $12 \mu \mathrm{m}$, the gas flow separation efficiency is $99.9 \%$ at any value of the wall thickness of I-beam elements within the range of $0.5-1.5 \mathrm{~mm}$. (Fig. 5).

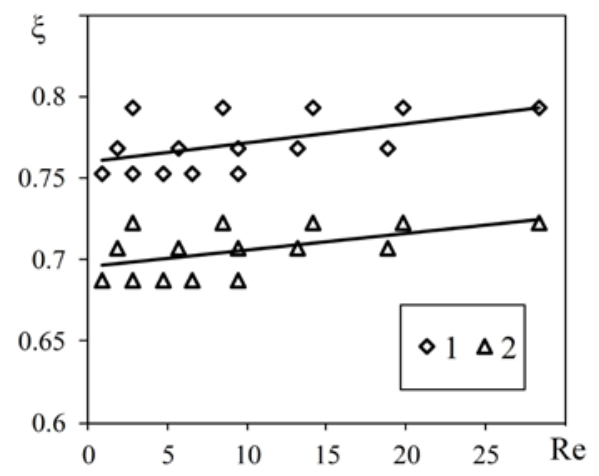

Fig. 6. Change in the hydraulic resistance coefficient from the Reynolds number at the following thickness of I-beam elements $\delta, \mathrm{mm}: 1-1 ; 2-1.5$. 
The hydraulic resistance coefficient of the first stage of a rectangular separator at the gas flow rate in the range of $3-7 \mathrm{~m} / \mathrm{s}$ on average is equal to 0.76 and 0.71 for the devices with thickness of I-beam elements of 1 and $1.5 \mathrm{~mm}$ respectively (Fig. 6). The maximum percentage error for this research is $3.89 \%$.

\section{Conclusion}

The use of a rectangular separator can be an alternative to the use of a cyclonic separator. Especially, rectangular separator is relevant to be used for gas purification from the finely dispersed particles with a diameter of up to $10 \mu \mathrm{m}$. In the course of research, it was found that the thicknesses of I-beam elements within the separator and inlet rate significantly affected the gas flow separation efficiency. The maximum efficiency of the rectangular separator is achieved at the wall thickness of $\delta=1 \mathrm{~mm}$ and inlet rate of $W=5 \mathrm{~m} / \mathrm{s}$. Advantages of the rectangular separator are low metal consumption, high efficiency of the gas flow separation and compactness.

The study was performed with funding from the RF President's grant project No. MK-4522.2018.8.

\section{References}

1. P. Baltrenas, M. Pranskevicius, A. Venslovas, Energy Procedia, 72, 188-195 (2015)

2. S. Altmeyer, V. Mathieu, S. Jullemier, P. Contal, N. Midoux, S. Rode, J.P. Leclers, Chem. Eng. Prog, 43, 511-522 (2004)

3. S.M. Clifford, J. Zhang, T. Sigsgaard, M. Jantunen, P.J. Lioy, R. Samson, M.H. Karol, Environ. Health Perspect., 115, 958-964 (2007)

4. L.Y. Hu, L.X. Zhou, J. Zhang, M.X. Shi, AIChE Journal, 51(3), 740-749 (2005)

5. I. Jakstoniene, S. Konoverskyte, P. Vaitiekunas, Užvalgos, 2(3), 141-149 (2009)

6. O.S. Makusheva, A.V. Dmitriev, N.A. Nikolaev, Chem. Pet. Eng., 46, 5-6, 330-333 (2010)

7. I.N. Madyshev, O.S. Dmitrieva, A.V. Dmitriev, MATEC Web Conf., 91, 01019 (2017)

8. A.V. Dmitriev, I.N. Madyshev, O.S. Dmitrieva, A.N. Nikolaev, Ecology and Industry of Russia, 21, 3, 12-15 (2017)

9. F. Shuanshi, X. Wang, X. Lang, Y. Wang, Nat. Gas Ind., 4(6), 470-476 (2017)

10. B. Sagot, A. Forthomme, L. Ait Ali Yahia, G. De La Bourdonnaye, J. Aerosol Sci., 110, 53-69 (2017)

11. A. Avci, I. Karagoz, J. Aerosol Sci., 53, 937-955 (2003)

12. S. Bernardo, M. Mori, A.P. Peres, R.P. Dionisio, Powder Technol., 162, 190-200 (2006)

13. A.V. Dmitriev, V.E. Zinurov, O.S. Dmitrieva, Vu L. Nguyen, Proceedings of Irkutsk State Technical University, 22, 3, 138-144 (2018) 